\title{
Academic Entitlement Scale: Development and Preliminary Validation
}

\author{
David T. Wasieleski, Mark A. Whatley, Deborah S. Briihl, Jennifer M. Branscome \\ Valdosta State University, Valdosta, USA
}

\begin{abstract}
Validity evidence was gathered for a new measure of academic entitlement. Two factors emerged during the creation of the scale - academic narcissism and academic outcome. Academic narcissism appears to reflect an expectation of high academic success unrelated to the effort or ability of the students. As expected, this factor was negatively correlated with students' self-reported GPA (grade point average). Males had higher academic narcissism scores than women. Academic outcome appears to reflect students' mindset that credit should be given for any effort, including attending class. As expected, this factor was not correlated with GPA and did not show sex differences. Implications and practical use of the scale are discussed.
\end{abstract}

Keywords: AE (academic entitlement), narcissism, academic attitudes, achievement

\section{Introduction}

A 2012 article in Education Week posed the question, "Are you enabling 'academic entitlement' in students?" (Sparks, 2012). This provocative article discussed the concept of AE (academic entitlement) and recent research conducted by Kopp, Zinn, Finney, and Jurich (2011). Kopp et al. (2011) are among a handful of researchers who have attempted to define and assess academic entitlement since the topic was formally introduced 20 years ago (Morrow, 1994). Since that time, increased attention has been given to AE, although little empirical data exist on the topic.

The underlying idea of AE is that students feel entitled to special treatment (e.g., extra credit, higher grades, ability to turn in work late, special exceptions, immediate access to instructors) when it is undeserved (Reinhardt, 2012). An additional theme is the expectation for a certain grade (usually high) that is not commensurate with either the effort expended or the quality of work (Reinhardt, 2012). Although common themes have emerged in conceptualizations of $\mathrm{AE}$, significant variability exists among operational definitions of $\mathrm{AE}$.

The inconsistency in conceptualizing and defining $\mathrm{AE}$ is one issue that impacts the accurate measurement of the construct. The literature discusses four measures of AE; however, all vary in how they attempt to assess AE (e.g., Chowning \& Campbell, 2009; Kopp et al., 2011). Definitions include references to elements such as "personal responsibility" (Chowning \& Campbell, 2009, p. 982) and "expectations of positive outcomes" (Kopp et al., 2011, p. 105). Other definitions include reference to the expectation of academic success unrelated to the quality of the product produced (e.g., paper, performance piece) or actual effort expended (e.g.,

David T. Wasieleski, Ph.D., Department of Psychology, Valdosta State University.

Mark A. Whatley, Ph.D., Department of Psychology, Valdosta State University.

Deborah S. Briihl, Ph.D., Department of Psychology, Valdosta State University.

Jennifer M. Branscome, Ph.D., Department of Psychology, Valdosta State University. 
Greenberger, Lessard, Chen, \& Farruggia, 2008). Yet, other authors discuss AE without defining it at all (Ciani, Summers, \& Easter, 2008).

The definition proposed by Reinhardt (2012) encapsulates the common threads among the various definitions of AE. In this definition, AE includes beliefs that rewards are deserved even though not supported by actual academic achievement, a lowered sense of personal responsibility for one's own academic achievement, unreasonable or unrealistic expectations of instructors, and overly demanding student attitudes and behaviors. This definition holds promise and incorporates many common-sense elements of AE although we propose an additional element: narcissism.

Narcissism is a trait in which individuals demonstrate dysfunctional behavior in self-appraisal and interpersonal relationships (Lukowitsky \& Pincus, 2013). Narcissistic characteristics range along a continuum from mild to severe (Foster, Campbell, \& Twenge, 2003). At the most severe or disordered level (i.e., narcissistic personality disorder), individuals will exhibit narcissistic behaviors across settings (e.g., home, work, school) (APA (American Psychiatric Association), 2013). Narcissistic characteristicstypically first manifest in early adulthood although traces of dysfunction can often been seen in childhood and adolescence. Narcissistic characteristics also are reported much more often in males than in females at the disordered (e.g., APA, 2013) and non-disordered levels (Bushman \& Baumeister, 1999). In one study, for example, researchers assessed narcissism in a group of 3,445 participants (Foster et al., 2003). In that study, males scored significantly higher on a measure of narcissism than females, even when age and reported income levels were controlled. These results are consistent with other studies' findings that men display more narcissistic characteristics than women (e.g., Joubert, 1998).

Those who are high on narcissism typically have an excessive need for admiration yet possess a significant lack of empathy (APA, 2013). An additional feature of narcissism is an unduly inflated or grandiose sense of self, even in the absence of special skills, abilities, or actual effort (APA, 2013). The narcissist typically rates his or her abilities more highly than is warranted and will correspondingly underestimate the accomplishments and achievements of others. Underlying these estimations is the assumption that others also view the narcissist as superior or special. Those high in narcissism typically aggrandize achievements or accomplishments, and are perceived often as arrogant, boastful, and pretentious.

A key aspect of the grandiose sense of self is a general sense of entitlement. The perception of superiority engenders a strong sense of general entitlement in which special treatment is expected. The person high in narcissism holds unreasonable expectations of special or favorable treatment and also assumes that others will comply without question (APA, 2013). The feelings of entitlement, coupled with a lack of empathy, frequently leads to the narcissist taking advantage of others to get what he or she wants. This would not be of concern, however, as narcissists firmly believe they are superior and therefore deserving of special treatment, even to the detriment of others. This sense of entitlement is typically observed in all, or most, settings (APA, 2013).

Researchers have hypothesized AE to be a subset of general entitlement and to be of increasing concern, as the prevalence of narcissism is increasing in college students (e.g., Twenge \& Foster, 2010). With AE, the core traits of narcissistic attitudes and behaviors are expected to manifest in a variety of setting-specific types of behaviors (e.g., demanding a high grade for poor quality work). As related to AE, the expectation of special treatment based on an inflated sense of self has been hypothesized to contribute to students' expectations of high academic success unrelated to the quality of the product produced or the actual effort produced by the student (e.g., Greenberger, Lessard, Chen, \& Farruggia, 2008). 


\section{Measurement of Academic Entitlement}

Although there is overlap and partial agreement between conceptualizations of $\mathrm{AE}$, significant differences exist in how $\mathrm{AE}$ has been assessed to date. Without a consistent and empirically-based operational definition of $\mathrm{AE}$, the creation of valid measurement instruments and prediction are impossible. To date, attempts to study $\mathrm{AE}$ and its attendant attitudes and behaviors have not progressed beyond the scale development stage and those possess considerable variance in the definition of the construct. In order to review the various contrasting conceptualizations of the construct, we provide an overview of AE scales.

\section{Achacoso (2002)}

Achacoso's 2002 measure of AE consists of 12 items, identified from an initial pool of 50 items (Achacoso, 2002). These 12 items compose two proposed factors of AE: entitlement beliefs and entitlement actions (Achacoso, 2002). Entitlement belief items assessed students' beliefs that they deserved special treatment or should achieve at a high level regardless of effort or quality of product (e.g., putting in minimal effort to learn material for class). Entitlement actions assessed the behaviors an entitled student would be expected to perform, such as arguing with a professor for more points on a test (Achacoso, 2002). Although the entitlement belief items clearly tap problematic student attitudes, what the entitlement action items assess is not as clear. Although some items reflect clearly inappropriate behavior no matter the situation (e.g., telling an instructor to give extra credit) others reflect behaviors that can be considered inappropriate or appropriate, depending on context, such as negotiating a grade with an instructor (Achacoso, 2002). Additionally, some items do not appear to address attitudes or behaviors that are solely reflective of AE.

\section{Chowning and Campbell (2009)}

Chowning and Campbell's (2009) scale of AE consists of 15 items and also yields two factors. The factors identified by Chowning and Campbell were externalized responsibility and entitled expectations. Externalized responsibility items assess the level of responsibility a student takes for his or her own academic success. Entitled expectations items assess students' expectations of their courses and professors (Chowning \& Campbell, 2009). Although a promising measure, the Chowning and Campbell scale, suffers from problems common to many scales, including double-barreled items, a potential acquiescence bias, and a low number of items (e.g., only five on entitled expectations).

\section{Greenberger et al. (2008)}

Greenberger et al. (2008) developed a scale of AE comprised of 15 items. The scale was based on the assumption that $\mathrm{AE}$ entails a sense of feeling that one deserves more and is entitled to more than others (Greenberger et al., 2008). Although the internal consistency of the scale is good ( $\alpha=0.87$ ), the structure of the scale was not assessed (Greenberger et al., 2008). Therefore, we are unable to determine if this scale reflects a unidimensional factor or if other factors are warranted. Additionally, some items appear to assess the idea that students do not distinguish well between level of effort and expected achievement (e.g., attending class often should equate to a good grade) and others that students should be accommodated at times, such as providing course notes to student (Greenberger et al., 2008). Also, a variety of behaviors are addressed by the items; however, some items do not appear to clearly measure AE beliefs specifically, such as an instructor should not be upset if a student takes a call in class (Reinhardt, 2012). Additional research should be conducted to assess the appropriateness of each item as well as the structure of the scale. 


\section{Kopp et al. (2011)}

Kopp et al. (2011) developed a scale based on five key factors proposed to underlie AE. The first factor reflects the idea that KR (knowledge) is a "right" that should be provided to a "customer" (i.e., student) with minimal effort or unpleasantness on the customer's part. The second factor addresses the idea that OP (other people) will provide all education necessary. PL (problems in learning) is the third facet and addresses the idea that any difficulties in learning are due to teacher inadequacy, issues with the course, or systemic issues rather than a student's lack of ability, skills, efforts, or work quality. SC (student control) is the fourth facet and reflects the idea that students hold a belief that they should have control over the policies of the course. The final facet concerns the idea that because a student has paid tuition, he or she believes that DT (certain outcomes) (e.g., good grades) are deserved (Kopp et al., 2011). By constructing the scale based on this theoretical conceptualization of $\mathrm{AE}$, the authors propose that the commonly identified aspects of $\mathrm{AE}$ are assessed while adding an additional dimension reflecting the concept of the student as a customer (Kopp et al., 2011). This scale provides an intriguing theoretical model upon which to base a measure of AE and preliminary analyses indicate good validity. However, additional validation of this scale is needed. Also, it would be of interest to address the trait of narcissism and how it relates to the five factor conceptualization proposed by the authors.

\section{Current Study}

Because of the significantly variability in the measurement and conceptualization of $\mathrm{AE}$ in existing scales, it is unclear if $\mathrm{AE}$ is being appropriately or accurately assessed. The purpose of this study, therefore, was to develop and conduct a preliminary validation of a scale of AE. Development of this scale built upon and expanded past efforts to define and measure AE, while attempting to provide a more definitive and empirically-based construction of the concept of AE.

\section{Method}

\section{Participants}

The participants were 63 male and 201 female undergraduates haphazardly selected fromValdosta State University during the 2011-2012 academic year. They ranged in age from 18-45 ( $M=19.96, S D=3.01)$. The present sample was 53.0\% White, non-Hispanic, 40.2\% African American, 2.3\% Hispanic, 1.9\% Asian, 0.4\% American Indian, and $2.3 \%$ indicated other. The academic classification of the sample was $40.9 \%$ freshman, $41.3 \%$ sophomore, $6.4 \%$ junior, $11.0 \%$ senior, and $0.4 \%$ indicated graduate student status. The average self-reported GPA (grade point average) was $3.10(S D=0.53)$ with 3.09 being the median.

\section{Materials}

An item pool consisting of 125 statements was generated to reflect academic entitlement attitudes. Sample items included "I often do other work in class", "I don't complain about the grades that I receive because I know that I deserve that grade", and "I should not be penalized for handing in late assignments". Participants rated their level of agreement with each item on a seven point scale from (1) "Strongly disagree" to (7) "Strongly agree". Positively worded items are reverse scored so that higher scores indicate more positive attitudes toward academic entitlement.

\section{Procedure}

Participants completed the 125 item survey in small groups $(<15)$. They were told the purpose of the 
study was to examine how students felt about academic entitlement.

Instructions to participants: After completing the demographic and items to be used as an initial test of construct validity, subjects read a brief paragraph at the top of the first page of the 125 item survey. Participants read:

Please read each item carefully and consider how you feel about each statement. There are no right or wrong answers to any of these statements, so please give your honest reactions and opinions. Please read each statement carefully, and respond by using the following scale:

The seven point rating scale was presented below these instructions and at the top of subsequent pages. Once participants completed the survey, they were thanked and informed how to obtain the results of the study.

\section{Results}

\section{Overview of Factor Analysis}

The data were subjected to an exploratory factor analysis using SPSS version 20.0. Factor analysis is a statistical technique enabling researchers to determine the main factors or clusters that underlie the relationship among a set of observed variables (Pedhazur \& Schmelkin, 1991; Tabachnick \& Fidell, 1989, 2001). The observed variables, for example the items comprising a scale, are inferred to indicate the unobservable or latent construct of interest (i.e., the factor). In the role of scale development, factor analysis is generally utilized to select sets of items that are indicative of the underlying construct the researcher is trying to assess (e.g., Glick \& Fiske, 1996). The objective of factor analysis is to discern the minimum number of factors that are compatible with the data.

The factor analysis used the maximum likelihood method of extraction and varimax rotation. The maximum likelihood method of extraction (Joreskog \& Lawley, 1968; Lawley \& Maxwell, 1963) generated the parameter estimates. Maximum likelihood estimates the population values for the determinants that maximize the probability of sampling the observed correlation matrix from a population (Tabachnick \& Fidell, 1989). Essentially, maximum likelihood minimizes the discrepancy between the population and sample covariance matrix, thereby maximizing the fitting function. Varimax rotation maximizes the factor loading variance and is the most commonly used method (e.g., Tabachnick \& Fidell, 1989).

\section{Factor Analytic Results}

We examined each item in regard to normal distribution, intercorrelations, skewness, kurtosis, and inter-item correlations. The Kaiser-Meyer-Olkin Measure of Sampling Adequacy and Bartlett's Test of Sphericity was examined to assess the suitability of the data for factor analysis. The Kaiser-Meyer-Olkin Measure of Sampling Adequacy was 0.72, which exceeded the 0.50 minimum requirement. Bartlett's Test of Sphericity revealed that there was a connection between the variables, thus permitting the factor analysis $\left(\chi^{2}(264)=16074.93, p<0.001\right)$.

The results indicated 39 factors with eigenvalues greater than 1.0. An eigenvalue is a measure of the variance accounted for by a given factor (Pedhazur \& Schmelkin, 1991; Tabachnick \& Fidell, 1989). After inspection of the scree plot, a two-factor solution consisting of 13 items per factor was deemed appropriate and allowing greater flexibility in research applications. The overall reliability (internal consistency) of the scale was $0.83(M=86.55, S D=18.78)$. Factor loadings and items are presented in Table 1 . 
Factor I $(M=27.54, S D=11.58$; Items 1-13) was labeled "academic narcissism" and accounted for $13.65 \%$ of the variance. The name of the factor reflects students' mindset that by receiving a grade lower than they believethey deserve is an insult to their intelligence. The coefficient of variation for Factor I was 0.42 . The coefficient of variation allows a comparison and assessment of the amount of variation that exists in a measure (Howell, 1992). The higher the value the more variation exists, and the greater the variation the greater the ability of a measure to discriminate between groups. The reliability (internal consistency) for the items comprising Factor I was 0.86.

Table 1

Attitudes Toward Academic Entitlement Scale Items and Factor Loadings

\begin{tabular}{|c|c|c|}
\hline \multicolumn{3}{|l|}{ Factor Loadings } \\
\hline Items & Factor I & Factor II \\
\hline 1. If a professor provides the PowerPoint slides on-line, I should not have to take additional notes. & 0.425 & 0.154 \\
\hline 2. I am disappointed in myself when I get a lower than expected grade. ${ }^{*}$ & 0.547 & 0.280 \\
\hline 3. Professors give me lower grades, because they feel threatened by my ability. & 0.634 & 0.024 \\
\hline 4. Universities should restrict the number of A's a professor can give. & 0.663 & 0.054 \\
\hline 5. Students should be allowed to take exams when it's convenient for them. & 0.566 & 0.166 \\
\hline 6. I will lie to get what I want. & 0.522 & 0.208 \\
\hline 7. Sometimes I get too busy to do my project, so it is OK is someone doesit for me & 0.567 & 0.106 \\
\hline 8. I usually just pop in to see a professor whenever-I don't really look at office hours. & 0.467 & 0.070 \\
\hline 9. I make sure to closely follow the requirements from the class. ${ }^{*}$ & 0.635 & 0.179 \\
\hline 10. It is my responsibility to know about any upcoming assignments. ${ }^{*}$ & 0.477 & 0.032 \\
\hline 11. I always make sure I let the professor know if I cannot make an appointment. ${ }^{*}$ & 0.544 & 0.118 \\
\hline 12. Course prerequisites are for people not as smart as me. & 0.548 & 0.027 \\
\hline 13. I feel as if I deserve more breaks than others because of my life issues. & 0.600 & 0.053 \\
\hline 14. I feel that my college should make sure I succeed academically. & 0.050 & 0.431 \\
\hline 15. I feel that effort should be worth points in my courses. & 0.101 & 0.600 \\
\hline $\begin{array}{l}\text { 16. I feel that because I am paying for my education, I should receive a high level of service } \\
\text { from my professors. }\end{array}$ & 0.175 & 0.567 \\
\hline 17. Professors who grade hard annoy me. & 0.018 & 0.576 \\
\hline $\begin{array}{l}\text { 18. If I go to a professor's office with a question, he/she should stop what they are doing and } \\
\text { answer me. }\end{array}$ & 0.073 & 0.498 \\
\hline 19. Professors should teach to the test. & 0.053 & 0.522 \\
\hline 20. If I have a borderline grade in a class, then the professor should give me the higher grade. & 0.128 & 0.546 \\
\hline 21. If another student gets a break, then I deserve to get a break no matter what the reason. & 0.188 & 0.525 \\
\hline 22. Everything should be discussed in class; I shouldn't have to figure out material in the book. & 0.142 & 0.619 \\
\hline 23. I should get some points for handing in an assignment. & 0.041 & 0.483 \\
\hline 24. All classes should offer extra credit. & 0.052 & 0.611 \\
\hline 25. If I come to class every time, I should pass the class. & 0.080 & 0.471 \\
\hline 26. Too many questions on tests are just trivial bits of information. & 0.138 & 0.505 \\
\hline
\end{tabular}
Note. Items marked with an “*” are reverse scored.

Factor II $(M=58.45, S D=14.07$; Items 13-26) was labeled "academic outcome" and accounted for $8.53 \%$ of the variance. The name of the factor reflects students' mindset that they should receive credit for any effort, including attending class. The coefficient of variation for Factor II was 0.23 and the reliability (internal consistency) was 0.84 . 


\section{Additional Analyses}

The 26 item Academic Entitlement Scale was subjected to ANOVAs (a one-way analyses of variance) to examine sex and ethnicity differences in overall scores and a correlational analysis examining the relationship between academic entitlement and GPA. For each analysis, an alpha level of 0.05 was used for all statistical tests and $r$ was calculated as the effect size (Rosenthal, 1991).

\section{Sex Differences in Academic Entitlement Attitudes}

A one-way analysis of variance was calculated on male and female participants' academic entitlement attitudes to determine whether sex differences exist in Factor I and Factor II.

Factor I. Male participants held stronger academic entitlement attitudes $(M=31.33, S D=13.27)$ than did female participants $(M=26.35, S D=10.75), F(1,261)=9.17, p=0.003(r=0.18)$.

Factor II. Male participants held about the same academic entitlement attitudes $(M=58.00, S D=13.13)$ than did female participants $(M=58.60, S D=14.38), F(1,262)=0.09, p=0.769(r=0.02)$.

\section{GPA and Academic Entitlement Attitudes}

Factor I. Students' self-reported GPA correlated negatively with academic entitlement attitudes. Students with higher GPA's endorsed weaker academic narcissism attitudes, $r(252)=-0.16, p=0.012$.

Factor II. Students' self-reported GPA did not correlate significantly with academic entitlement attitudes, $r(253)=-0.07, p=0.299$.

\section{Discussion}

The goal of this study was to develop a psychometrically sound measure that would provide researchers with a tool to study academic entitlement attitudes. The current effort to define a valid construct of college student academic entitlement has resulted in a preliminary two-factor scale. The current study will provide preliminary validation through correlation with self-reported GPA.

There are several positive aspects associated with the development of this scale. First, the scale is a multidimensional measure which aids in the interpretation of such attitude differences. This adds to, and furthers, our understanding of academic entitlement. Second, the scale has respectable reliability. Third, the initial assessment of construct validity was positive, in that the scale related to other variables in predicted ways (e.g., Pedhazur \& Schmelkin, 1991). Fourth, the scale has a high coefficient of variation, particularly for the factor of academic narcissism, allowing the scale to discriminate between groups when such groups differ on academic entitlement attitudes (e.g., Howell, 1992). Lastly, the scale is relatively brief allowing more versatility in research and professional settings. Cross-validation is the next step, followed by further testing of the scale through correlation with academic behaviors such as studying habits, class attendance, and grades.

For this study, we proposed adding narcissism to the existing conceptualizations of AE. The essential features of narcissism are an inflated sense of self-worth, excessive need for admiration, and a lack of empathy (APA, 2013). Narcissism involves anentrenched sense of entitlement based upon an unrealistically high evaluation of a person's skills and abilities. Those high in narcissism believe themselves to be special and expect unending admiration as a result. In the academic setting, the expectation of special treatment has been proposed as an explanation of students' expectations of high academic success unrelated to the effort or ability of the students. Our analyses suggest that narcissistic characteristics underlie attitudes and behaviors of AE.

The results of this study revealed that male participants held stronger academic entitlement attitudes than 
did female participants. This finding is consistent with Ciani, Summers, and Easter (2008) that found males engage in a self-perception process that results in a greater sense of entitlement in classroom settings. Academics is one area where the comparison of achievement is robust. The tendency for people to react more favorably to comparison information indicating they have performed better than other ingroup members appears to be strongest among idiocentrics who value competition and "getting ahead" of others and attenuated among allocentrics who value ingroup harmony and "getting along" with other ingroup members (Triandis, 1995; Wolfe, Lennox, \& Cutler, 1986). The focus on achievement comparisons may be more important to males as research indicates that males tend to be more idiocentric than females, and females tend to be more allocentric than males (Grace, 2001). Because idiocentrics give more weight to being independent and superior to others compared to allocentrics, one plausible outcome is that males would be react more strongly to achievement comparisons compared to females (Festinger, 1954; McFarland \& Buehler, 1995; Triandis, 1995). By focusing on achievement comparisons, males are more likely to gain information that may allow them to develop superiority over others. Males appear to be concerned with "getting ahead" of others (see Arkin, 1981; Wolfe, Lennox, \& Cutler, 1986).

The apparent divergence between idiocentrics' and allocentrics' desire to "get ahead" or "get along" would appear to lead to differential attention to the social comparison of achievement. Because idiocentrics value competition, being independent, and being superior to others to a greater extent than do allocentrics, idiocentrics should be more concerned with achievement comparisons than allocentrics (Festinger, 1954; Schwartz, 1994; Triandis, 1995). This predisposition is one plausible reason why male subjects in the current study scored higher on the academic narcissism factor compared to female subjects (see Foster, Campbell, \& Twenge, 2003). The initial evidence reported here suggests the basic fundamental difference in how males and females perceive their immediate social world moderates the academic entitlement attitudes.

Although the idea of entitlement as applied to the academic setting is appealing, the relationship between $\mathrm{AE}$ and entitlement as a trait is unknown. It is not clear if AE truly is a subtype of characterological entitlement or if the academic setting is a catalyst to the development of entitled attitudes and behaviors. If AE is a subset of entitlement (as seen in narcissism), a reasonable expectation is that students high on AE will demonstrate entitled attitudes and behaviors across settings. If $\mathrm{AE}$ is a function of the academic setting, then entitled attitudes and behaviors would occur only in that setting and would not manifest in other settings. Furthermore, if $\mathrm{AE}$ is specific to the academic setting, it would be valuable to understand what influences its expression in the setting (e.g., competitive structure), the person (e.g., idiocentrics), or both. By addressing these questions, a clearer picture of the construct of AE will emerge.

Because there have been relatively few studies concerning attitudes toward academic entitlement, further research needs to be conducted, regionally and cross-culturally, to examine differences in academic entitlement trends and characteristics. For example, individuals from the Southern part of the United States tend to adhere more strongly to traditional roles and attitudes (e.g., Conlee, 2012; Lublin \& Brewer, 2003; Rice \& Coates, 1995; Secret, 1987) as well as being socialized in to a culture of honor (Nisbett \& Cohen, 1996). Because socialization can vary as a function of region and culture, we should not be surprised that various psychological constructs, such as academic entitlement and narcissism, vary as well (Foster et al., 2003).

Additionally, because of the lack of attention given to the area, the information derived from this scale could be used to examine other areas for meaningful and important differences. For example, as college campuses become more diverse, one would need to be aware of cross-cultural differences in attitudes as they 
relate to academic entitlement. For example, individualistic cultures (e.g., United States, Australia) have been shown to differ from collectivistic cultures (e.g., Japan, China, Mexico) in important ways. For example, individualists emphasize personal fate, personal achievement and independence; whereas, collectivists emphasize group fate, group achievement, and interdependence within the group (Triandis, 1995). The characteristics of individualism appear to predispose those raised in individualistic cultures to narcissism as those living in individualistic societies scored higher on a measure of narcissism compared to those living in collectivistic societies (e.g., Foster et al., 2003). As such, students from individualistic cultures may endorse stronger academic entitlement attitudes as a function of narcissistic tendencies compared to students from more collectivistic cultures (e.g., Foster et al., 2003).

An empirically-based measure of AE has the potential to be useful in a variety of academic settings such as student success centers, academic affairs departments, and at the classroom level. Academic entitlement has been demonstrated to be associated with negative consequences such as lower grade point averages and increased engagement in academically dishonest behavior (van Wieirngen, Formicola, Peirone, \& Falsetta, 2013). Academic entitlement has also been found to be a predictor of feelings of entitlement in the workplace as well as unrealistic beliefs or expectations about promotions, bonuses, salaries, and workplace conditions (vanWieirngen, Formicola, Peirone, \& Falsetta, 2013). By revealing a student's beliefs about academic entitlement, the student may be able to learn to adjust his or her behavior to positively impact success. With careful and reasoned use, knowledge of a student's AE beliefs and attitudes can also provide valuable information to assist students in developing effective and functional strategies for success, in and out of the classroom, potentially allowing for increased social, academic, and professional success.

\section{References}

Achacoso, M. V. (2002). "What do you mean my grade is not an A?" An investigation of academic entitlement, causal attributions, and self-regulation in college students (Unpublished doctoral dissertation, University of Texas, Austin).

APA (American Psychiatric Association). (2013). Diagnostic and statistical manual of mental disorders (5th ed.). Washington, D.C.: Author.

Arkin, R. M. (1981). Self-presentation styles. In J. T. Tedeschi (Ed.), Impression management theory and social psychological research (pp. 311-333). New York: Academic Press.

Bushman, B. J., \& Baumeister, R. F. (1999). Threatened egoism, narcissism, self-esteem, and direct and displaced aggression: Does self-love or self-hate lead to violence? Journal of Personality and Social Psychology, 76, 367-376.

Chowning, K., \& Campbell, N. J. (2009). Development and validation of a measure of academic entitlement: Individual differences in students' externalized responsibility and entitledexpectations. Journal of Educational Psychology, 101, 982-997.

Ciani, K. D., Summers, J. J., \& Easter, M. A. (2008). Gender differences in academic entitlement among college students. The Journal of Genetic Psychology, 169, 332-344.

Conlee, D. (2012). Gender roles in organizations in the Southeastern United States (Master's thesis, Gonzaga University, Spokane, Washington).

Festinger, L. (1954). A theory of social comparison processes. Human Relations, 7, 117-140.

Flouri, E. (2006). Parental interest in children's education, children's self-esteem and locus of control, and later educational attainment: Twenty-six year follow-up of the 1970 British Birth Cohort. British Journal of Educational Psychology, 76(1), 41-55. doi:10.1348/000709905X52508

Foster, J. D., Campbell, W. K., \& Twenge, J. M. (2003). Individual differences in narcissism: Inflated self-views across the lifespan and around the world. Journal of Research in Personality, 37(6), 469-486. doi:10.1016/S0092-6566(03)00026-6

Glick, P., \& Fiske, S. T. (1996). The ambivalent sexism inventory: Differentiating hostile and benevolent sexism. Journal of Personality and Social Psychology, 70, 491-512. 
Grace, S. L. (2001) The nature of independent and interdependent self-construals: A focus on psychological relatedness. (Electronic Theses and Dissertations, Paper 4115). Retrived from http://scholar.uwindsor.ca/etd/4115

Greenberger, E., Lessard, J., Chen, C., \& Farruggia, S. P. (2008). Self-entitled college students: Contributions of personality, parenting, and motivational factors. Journal of Youth and Adolescence, 37, 1193-1204. doi: 10.1007/s10964-008-9284-9

Howell, D. C. (1992). Statistical methods for psychology (3rd ed.). Boston, M.A.: PWS-Kent Publishing.

Joreskog, K. G., \& Lawley, D. N. (1968). New methods in maximum likelihood factor analysis. British Journal of Mathematical and Statistical Psychology, 21, 85-96.

Joubert, C. E. (1998). Narcissism, need for power, and social interest. Psychological Reports, 82, 701-702.

Kopp, J. P., Zinn, T. E., Finney, S. J., \& Jurich, D. P. (2011). The development and evaluation of theacademic entitlement questionnaire. Measurement and Evaluation in Counseling and Development, 44(2), 105-129. doi: $10.1177 / 0748175611400292$

Lawley, D. N., \& Maxwell, A. E. (1963). Factor analysis as a statistical method. London: Butter worths.

Lublin, D., \& Brewer, S. E. (2003). The continuing dominance of traditional gender roles in southern elections. Social Science Quarterly, 84(2), 379-396.

Lukowitsky, M. R., \& Pincus, A. L. (2013). Interpersonal perception of pathological narcissism: A social relations analysis. Journal of Personality Assessment, 95(3), 261-273. doi: 10.1080/00223891.2013.765881

McFarland, C., \& Buehler, R. (1995). Collective self-esteem as a moderator of the frog-pond effect in reactions to performance feedback. Journal of Personality and Social Psychology, 68, 1055-1070.

McKeachie, W. (1994). Teaching tips: Strategies, research, and theory for college and university teachers (9th ed.). Lexington, MA US: D C Heath.

Morrow, W. (1994). Entitlement and achievement in education. Studies in Philosophy and Education, 13, 33-47.

Nisbett, R. E., \& Cohen, D. (1996). Culture of honor: The psychology of violence in the South. Boulder, C.O.: Westview Press.

Pedhazur, E. J., \& Schmelkin, L. P. (1991). Measurement design, and analysis: An integrated approach. Hillsdale, N.J.: Lawrence Erlbaum Associates.

Reinhardt, J. (2012). Conceptualizing academic entitlement: What are we measuring? (Unpublished thesis, University of Windsor, Windsor, Canada).

Rice, T. W., \& Coates, D. L. (1995). Gender role attitudes in the Southern United States. Gender \& Society, 9(6), $744-756$.

Rosenthal, R. (1991). Meta-analytic procedures for social research. Newbury Park, C.A.: Sage Publications.

Schwartz, S. H. (1994). Beyond individualism and collectivism: New cultural dimensions of values. In U. Kim, H. C. Triandis, C. Kâgitçiba_i, S. C. Choi, \& G. Yoon (Eds.), Individualism and collectivism: Theory, method, and applications (pp. 85-122). Newbury Park, C.A.: Sage Publications.

Secret, P. E. (1987). The impact of region on racial differences in attitudes toward legal abortion. Journal of Black Studies, 17(3), 347-369.

Sparks, S. (2012, May 27). Are you enabling "academic entitlement" in students? (Web log). Retrieved from http://blogs.edweek.org/edweek/inside-school-research/2012/05/are_you_enabling_academic_enti.html

Tabachnick, B. G., \& Fidell, L. S. (1989). Using multivariate statistics (2nd ed.). New York: Harper \& Row Publishers.

Tabachnick, B. G., \& Fidell, L. S. (2001). Using multivariate statistics (4th ed.). Boston, M.A.: Allyn and Bacon.

Triandis, H. C. (1995). Individualism and collectivism. San Francisco, C.A.: Westview Press.

Twenge, J. J., \& Foster, J. D. (2010). Birth cohort increases in narcissistic personality traits among American college students. Social Psychological and Personality Science, 1(1), 99-106. doi: 10.1177/1948550609355719.

Twenge, J. M., Zhang, L., \& Im, C. (2004). It's beyond my control: A cross-temporal meta-analysis of increasing externality in locus of control, 1960-2002. Personality and Social Psychology Review, 8, 308-319.

vanWieirngen, K. A., Formicola, A., Peirone, A., \& Falsetta, T. (2013, May 30). Academic entitlement leads to negative outcomes, student researchers find. Retrieved from http://www.uwindsor.ca/dailynews/2013-05-29/academic-entitlement-leadsnegative-consequences-student-researchers-find

Wolfe, R. N., Lennox, R. D., \& Cutler, B. L. (1986). Getting along and getting ahead: Empirical support for a theory of protective and acquisitive self-presentation. Journal of Personality and Social Psychology, 50, 356-361. 